\title{
Designing a vaccine for tuberculosis
}

\section{Unravelling the tuberculosis genome — can we build a better BCG?}

Tuberculosis is a disease of superlatives. Mycobacterium tuberculosis causes more deaths annually than any other infectious agent. ${ }^{1}$ Globally, it is one of the major pathogens associated with HIV disease. ${ }^{1}$ The tuberculosis vaccine, BCG, has been given to more people than any other vaccine. ${ }^{23}$ However, although this vaccine confers clear benefit against disseminated childhood tuberculosis, its efficacy against adult pulmonary disease has varied widely in different clinical trials. ${ }^{24}$ Curiously, protection induced by BCG seems to improve with increasing distance from the equator. ${ }^{2}$ In a large randomised controlled trial in Madras, southern India, and a large observational study in Malawi, BCG was no better than saline. ${ }^{35}$ It would be good to do better.

The reasons for the failure of BCG in adults remain unclear. Indeed, immunity to tuberculosis is poorly understood both at a cellular and molecular level. ${ }^{16}$ It is possible that the ability of BCG to protect against initial infection may wane with time. Alternatively, BCG may be unable to prevent the establishment of dormant infection, so giving the potential for reactivation later in adult life. An ideal tuberculosis vaccine would be given at birth as a non-living subunit formulation (with a view to safety and quality control) and would confer lifelong protection. Possible alternative profiles for new vaccines include a "booster" vaccine that could be given to young adults (a high risk age group), a "transmission blocking" vaccine that would decrease positivity in sputum smears, and an "immunomodulating" or therapeutic vaccine ${ }^{7}$ that could be used as an adjunct to shorten current treatment protocols.

In March 1995, at a meeting in Madrid organised by the World Health Organisation's global programme for vaccines, groups from the public and private sectors met to discuss a global coordinated programme for developing vaccines. Recent progress in mycobacterial genetics has uncovered exciting new strategies for generating candidate vaccines. Scientists are beginning to understand the molecular basis of attenuation of BCG and other avirulent strains of tuberculosis. ${ }^{8} 9$ This raises the possibility of designing new live vaccines, either by inactivating key genes in $M$ tuberculosis ${ }^{10}$ or by adding new genes to BCG. For example, BCG has been constructed to express cytokines designed to enhance its immunogenicity. ${ }^{11}$ Another approach is based on developing a subunit vaccine. Vaccination with secreted antigens isolated from $M$ tuberculosis cultures has been shown to confer significant protection against challenge in experimental models. ${ }^{12}{ }^{13}$

Alternatively, genes encoding appropriate antigens can be delivered using suitable expression systems or vectors for immunisation. Promising results have been achieved by vaccination with nucleic acid or "naked DNA,"14 and a range of bacterial or viral vectors is also under consideration. With information from the tuberculosis genome project, currently under way at the Sanger Centre in Cambridgeshire, it is possible to consider screening all the genes of $M$ tuberculosis for vaccine efficacy rather than relying on selection of particular proteins from laboratory cultures. ${ }^{15}$

Research is under way to evaluate new candidate vaccines by protection studies in animals. Several animal models of tuberculosis have been established. Mice are relatively resistant to disease and fail to reproduce caseous necrosis, a hallmark of human tuberculosis. Nevertheless, mouse tuberculosis is a good starting point. ${ }^{6}$ The mouse model is easy to manipulate, and many reagents are available to help elucidate immune mechanisms. As a result, ironically, we know more about immunity to tuberculosis in mice than in humans. Alternative models, such as guinea pigs, rabbits, and non-human primates, ${ }^{16}$ are better mimics of particular aspects of human disease. However, such studies are expensive and restricted to a few specialised laboratories. A triage system has been established to screen new vaccine candidates for clinical evaluation. Initial testing is by parenteral challenge in the less expensive mouse model, with promising candidates going on for further evaluation in aerosol challenge protocols.

Formidable problems are likely in moving a vaccine from the laboratory into clinical trials. Protection in animal models cannot be taken as a measure of protection in humans. Seventy years of experience with BCG have shown the difficulty of evaluating a vaccine against tuberculosis. There is clearly a need to identify a short term surrogate marker of potential efficacy. The current Mantoux or Heaf tests based on skin test hypersensitivity do not reflect protection. ${ }^{17}$ Attempts are being made to develop new skin tests based on improved antigen preparations or using in vitro assays to assess protective, cell mediated immune responses. In the longer term, an ideal trial would involve vaccinating neonates and testing for protection against disease in young adults. However, initial shorter term trials of any new vaccine will probably focus on attempts to boost responses in high risk groups who may already have been exposed to infection or BCG vaccination.

Developing an improved tuberculosis vaccine presents a daunting task in the face of our limited understanding of the organism's virulence and the host's immune response. Given the superlative impact of tuberculosis, we cannot avoid this task. In fact we require superlative effort.

ADAM S MALIN

Clinical lecturer

Department of Clinical Sciences,

London School of Hygiene and Tropical Medicine,

London WC1E 7HT

DOUGLAS B YOUNG

Professor of medical microbiology

Department of Medical Microbiology,

Imperial College School of Medicine at St Mary's Hospital,

London W2 1 PG

1 Bloom BR, Murray CJ. Tuberculosis: commentary on a reemergent killer. Science 1992;257:1055-64.

2 Fine PE, Rodrigues LC. Modern vaccines. Mycobacterial diseases. Lancet 1990;335:1016-20.

3 Bloom BR, Fine PEM. The BCG experience: implications for future vaccines against tuberculosis. In: Bloom BR, ed. Tuberculosis: pathogenesis, protection and control. Washington: American Society for Microbiology, 1994:531-57.

4 Besnard $M$, Sauvion S, Offredo C, Gaudelus J, Gaillard JL, Veber F, et al. Bacillus Calmette-Guerin infection after vaccination of human immunodeficiency virus-infected children. Pediatr Infect Dis $f$ 1993;12:993-7.

5 Ponnighaus JM, Fine PE, Sterne JA, Wilson RJ, Msosa E, Gruer PJ, et al. Efficacy of BCG vaccine against leprosy and tuberculosis in northern Malawi. Lancet 1992;339:636-9.

6 Orme IM, Andersen P, Boom WH. T cell response to Mycobacterium tuberculosis. $f$ Infect Dis 1993;167:1481-97.

7 Stanford JL, Grange JM. New concepts for the control of tuberculosis in the twenty first century. $¥ R$ Coll Physicians Lond 1993;27:218-23.

8 Mahairas GG, Sabo PJ, Hickey MJ, Singh DC, Stover CK. Molecular analysis of genetic differences between Mycobacterium bovis BCG and virulent M. bovis. F Bacteriol 1996;178:127482.

9 Collins DM, Kawakami RP, De Lisle GW, Pascopella L, Bloom BR, Jacobs WR Jr. Mutation of the principal sigma factor causes loss of virulence in a strain of the Mycobacterium tuberculosis complex. Proc Natl Acad Sci U S A 1995;92:8036-40.

10 Reyrat JM, Berthet FX, Gicquel B. The urease locus of Mycobacterium tuberculosis and its utilization for the demonstration of allelic exchange in Mycobacterium bovis bacillus Calmette-Guerin. Proc Natl Acad Sci U S A 1995;92:8768-72.

11 Murray PJ, Aldovini A, Young RA. Manipulation and potentiation of antimycobacterial immunity using recombinant bacille Calmette-Guerin strains that secrete cytokines. Proc Natl Acad nity using recombinant bacil
Sci USA 1996;93:934-9.

12 Andersen P. Effective vaccination of mice against Mycobacterium tuberculosis infection with a soluble mixture of secreted mycobacterial proteins. Infect Immun 1994;62:2536-44.

13 Horwitz MA, Byong-Wha EL, Dillon BJ, Harth G. Protective immunity against tuberculosis induced by vaccination with major extracellular proteins of Mycobacterium tuberculosis. Proc Natl Acad Sci U S A 1995;92:1530-4.

14 Silva CL, Lowrie DB. A single mycobacterial protein (hsp 65) expressed by a transgenic antigen-presenting cell vaccinates mice against tuberculosis. Immunology 1994;82:244-8.

15 Tang DC, De Vit M, Johnston SA. Genetic immunization is a simple method for eliciting an immune response. Nature 1992;356:152-4.

16 Walsh GP, Tan EV, dela Cruz EC, Abalos RM, Villahermosa LG, Young LJ, et al. The Philippine cynomolgus monkey (Macaca fasicularis) provides a new nonhuman primate model of tuberculosis that resembles human disease. Nat Med 1996;2:430-6

7 Fine PEM, Sterne JAC, Ponnighaus JM, Rees RJW. Delayed-type hypersensitivity, mycobacterial vaccines and protective immunity. Lancet 1994;344:1245-9. 